\title{
Defining, maintaining and managing metadata standards
}

\begin{abstract}
Carol Owens
is Head of Media Data Group in the Consulting Services division of Siemens Business Services. Her team provides the BBC's metadata integration service, including the Standard Media Exchange Framework (SMEF) Data Model, and media asset management consulting. She worked for the BBC for 26 years, spending the first half of her career in film editing and (briefly) production, then moving into operational management. The last nine years were spent in developing strategic thinking about digital production technology and its impact on the business, culminating in the present work on metadata standards and asset management. She chaired the European Broadcasting Union's P/Meta Project and Metadata Advisory Group, and was made a Fellow of the Royal Television Society in November 2001.
\end{abstract}

Keywords: content-data architectures, requirements planning, vendor selection

Abstract Prospective buyers of media asset management (MAM) systems are taking unacceptable business risks if they do not clearly define their metadata requirements up front, and then apply appropriate methods to ensure that the delivered solution meets those requirements. In addition, the subsequent data management approach must be tailored to meet the business needs on an appropriate scale to ensure that expected benefits are fully realized.

\section{THE PROBLEM}

Q: When is a digital asset not an asset?

A: When you can't find it, don't know what's in it or what you can do with it.

That is a rather glib summary of one of the main problems to be solved in digital asset management (DAM) projects, which arises when insufficient, inaccurate or incomprehensible information is held about the digitized media objects. Simply digitizing video and audio does not turn them into assets: the value arises from the use the asset or system owner puts them to, and their effective use depends on the quality and relevance of the associated data.

In the professional media business, the data associated media essence is known as metadata, although the word has a different meaning in mainstream Information Systems circles. Media metadata includes items like title, episode, format, duration, or channel. It is crucial for prospective buyers and project managers to understand the importance of clearly defining their metadata requirements upfront, and then applying appropriate methods to ensure that the delivered solution meets those requirements.

The risks of not doing so are that the business usability of the system will be restricted to the supplier's view of user needs, potentially requiring disruptive changes to working practices and falling short on expected benefits. Alternatively, the gap will have to be filled post hoc by expensive fixes and human interpretation. At worst, the new system may cause 
new problems, or is simply digitizing an existing problem. These risks are multiplied by the complexity of the integration environment into which the DAM system must fit, and the user processes, which it must support.

The justification for a structured approach to metadata may start with a look at some business examples where it was not used.

\section{CASE STUDIES}

- A pilot project to test digital asset management technology was set up in a landmark television series. The series had UK and US versions, a schools derivative, a book and a website: it should have been a perfect candidate for DAM. All went well while a professional researcher entered and annotated each media file, but when the rest of the production team started to use the system, chaos ensued. There were no standard naming conventions, nor structured data items, and even the person who entered the data could not find it again.

- A broadcaster installed a leading-edge server-based media store to capture incoming video contributions for use in rolling news. The media managers entering data into the system identified each stored object by the source and time of receipt, and an ad hoc story name. The story name might change during the day, and other users often had to speak to the person who originally entered the data to see if they remembered the associations. As time passed, the chances of finding the material again progressively reduced.

- A television sports library had been setting up a new database to log video content for each major international sporting event; each of the 18 databases they set up was incompatible with the others and with the main library system. Any search for material covering an athlete's career history required expert searching across five or six separate databases in turn by someone who already knew which events the athlete had competed in.

- A major broadcast archive received so much video material it was unable to view and catalog everything, so it relied on information delivered with the finished programs to create catalog entries for 90 per cent of its intake. This information was created by someone in the program team, often after the schedule had ended and the original research team had dispersed. There was little quality control, and during a period of budget reductions, many programs were delivered with no supporting information at all. The archive was unable to catalog them, let alone digitize them, and consequently they were unavailable for any further re-use.

All these examples demonstrate waste in terms of unproductive use of peoples' time, bottlenecks in the search and retrieval process, and lost opportunities to exploit media assets whether digital or old-fashioned analog.

\section{SCOPE}

The approach to metadata depends on the scope and scale of the user's ambition for DAM. Some buyers want a selfcontained system to support a clearly delimited set of business processes. Others see DAM as an element of supply chain integration in a larger organization, with multiple system interfaces and business hand-offs. There is a global level too, where DAM systems support international and inter- 
company trading or exchange with distributed management and control.

For the latter two options, the further consideration is whether data interoperability is achieved via automatic exchanges between systems, or whether human interpretation is expected. Human intervention may add value such as specialist knowledge, or it may just be a waste of effort occasioned by a failure of system integration. The larger the scale of the DAM environment, and the higher the degree of automation required, the greater the need for standardization and precision, eliminating ambiguity, and improving processes.

One of the biggest problems for data entry in digital asset management is that the person creating the data is rarely the end user who benefits from it. While the digital asset is work-in-progress and shared by a small number of people, it may be possible to function with scant or idiosyncratic data. However, as soon as access to the asset is extended across multiple users and/or over a long period of time, the quality, completeness and comprehensibility of the data become crucial. The challenge for DAM system implementers and business sponsors is to take a full lifecycle approach to metadata specification and management.

\section{METADATA DEFINITION}

The starting point is the logical definition of the data required to support all of the intended business processes. This definition takes two forms: first the identification of the items of data required by users to enable them to perform their business functions and workflow, and required by the system to perform its data management functions. The data items will represent things or concepts in use and their characteristics, eg a media object, its title and duration. The number of items is irrelevant; it can be large or small, but it must be enough to enable all users to do their work. This assumes of course that there is an understanding of what the system is to do, so that the metadata needed to do it with can be identified.

Having identified the data, the second activity is to provide clear and unambiguous names and definitions for the items, which all parties can agree. Rules will also be developed for the relationships and hierarchies between data items, which reflect relationships in the real world. For example, a program or editorial object can have one or many versions, but only one program version can occupy an individual transmission slot or publication event. This sort of logical information is best expressed using some standardized notation from the Information Systems world, which potential DAM suppliers and system integrators are likely to understand when presented with it, eg via an invitation to tender.

It is important to define metadata as part of the requirements process in order to ensure common understanding between users and systems. This is absolutely crucial when physical system integration requires automatic exchange or sharing of data without human interpretation: machines have only limited capability to deal with ambiguity, and if told that field 1 in database A always means the same as field 4 in database B, will operate accordingly, whether or not it is actually true.

Further, it is helpful to have a documented statement of the data requirements to assist in the selection of 
suitable solutions, and to identify any gaps, configuration issues, and potential work-arounds that may be needed. In the media industry, users often have only a partial awareness of their data (and other) requirements, and by presenting it in black-and-white, a formal analysis can offer a dramatic stepchange in their own appreciation of what they need to do. Common understanding can be a tough goal, when business areas use different terms for the same things or the same terms for different things, but sensitive data analysis can arrive at a view where local synonyms are allowed, and genuine difference is clearly expressed.

\section{METHODS}

Data analysts use various formal methods and documentary products to arrive at and express their conclusions. These are largely industry standard, and usually include some sort of diagrammatic representation or model, plus a detailed catalog of the terms and definitions used in it. These could consist of an entity relationship or class diagram, plus a data dictionary. There might further be interface specifications and exchange models.

If the DAM system to be procured is a package application, presenting only its interfaces to the user or integrator, the interface specification would be the most useful working document. However, for awareness of the overall business context, and when either bespoke development or detailed configuration is planned, it is best to have a full logical model, independent of technology. In addition, there should be process models; use cases if unified modeling language (UML) methodology is being used; and system context diagrams. The documents should be produced keeping in mind their purpose and target audience in mind, (eg whether business or technical).

As well as the items of data, which represent things or concepts and their characteristics, there are sets of values attached to the characteristics which need to be defined. These are known as reference data, and can, for example, be lists of country codes or cataloging terms, and can often be presented in systems as pick-lists. Some lists may be locally created and managed, while others are international standards such as ISO language codes. It is important to identify them clearly and make sure that any other systems in the data integration environment use the same lists as far as possible.

On the subject of international standards, it may be advisable to adopt a standard data scheme if the DAM system is to operate across international boundaries, or it is to use commodity equipment which conforms to a particular standard. There are various published options, such as the European Broadcasting Union's P_META Scheme and W3C's Dublin Core, but they are designed respectively for business-tobusiness media and metadata exchange, and first level inter-archive retrieval not for in-house workflow use.

Experience shows that every organization has specific local needs, which require local analysis and specification, but international standards can be integrated as appropriate.

\section{TECHNOLOGIES}

The primary technical requirement for metadata capability in DAM is the technology for capturing, storing and exchanging data. By data, this may 
include not only alphanumeric business data but also the digitized media essence itself, and any metadata embedded with it. International and de facto supplier-led standards dictate the physical coding of media and carriage of metadata in file and streaming formats. There is pressure in the industry for suppliers to adopt open standards in the interests of interoperability and exchange, but some still resist this in favor of proprietary formats or "enhanced versions" of international standards which cannot be supported by their competitors. This tends to create supplier lock-in once the initial buying decision has been made, although this is not necessarily in the client's long-term best interests.

Much emphasis has been placed in recent years on the development of the Material eXchange Format (MXF) for media interoperability and metadata carriage, but take-up is proceeding more slowly than expected. This may be due to the flexibility of the format specification, which perversely may be discouraging potential adopters who are spoilt for choice, or due to tensions between key suppliers which are causing proprietary aspects to creep in.

However, the cause is definitely worth pursuing if MXF can become an open element for exchange in an integrated data and DAM environment. The rest of the electronic alphanumeric data are generally stored in databases and may be exchanged using XML or other IT data formats.

The second technical requirement for metadata in DAM, and any other professional data management domain, is the use of appropriate tools for modeling and managing the data specifications. Data analysts use Computer Aided Software Engineering
(CASE) tools to create their diagrams and dictionaries, and manage the different versions. For enterprise data management teams, it may be useful to employ a central repository tool, which holds individual project data models and tracks their relationship with the overall corporate data model. This enables new development or integration projects to see immediately which data items and definitions they need for a particular business area, and what is already implemented in connecting systems.

\section{MANAGEMENT}

The scope of the DAM implementation as described earlier will also condition the approach to operational data management once the system has been specified and implemented. There are, however, some common factors. All users must be trained in data entry, and quality must be monitored. Data integrity must be assured through version control, clear identification of the master record if data are to be replicated, and regular scrutiny of data quality. There must also be people whose job and mission is to make this work for the business. In large-scale enterprise implementations there should be senior champions, operational managers and system administrators, and it is possible to establish these roles within a practice known as data stewardship.

For a self-contained system with a controlled user base it may be enough to have a system administrator and super user to enforce working practices and manage change control. For any integrated system where there is a great deal of data in play, covering a whole range of subjects and supporting a similar range of business processes, it 
will need considerably more. Particular subjects have key relevance to specific business areas, who will feel that they "own" the data to some extent. Good examples are data about personnel or publication schedules. The schedule owner will want to control the information in use, yet at the same time allow others to use it.

This responsibility may be embodied in the role of senior managing steward, who needs to agree that the data in his or her area is correctly defined according to business requirements, along with its necessary qualities such as accuracy and timeliness, and to approve its use in other areas or system developments. Any key reference data in this area should be approved and owned at this level, such as the set of names for publication channels, or the hierarchy of job grades in human resources (HR). At operational level, the steward will be more hands-on and look at data entry procedures, quality management, and change control. The system administrator will support these requirements in daily service delivery.

In a large and complex organization it may further be beneficial to establish an enterprise data management team which sets strategies, policies and standards, and assists individual projects and business areas to implement them for the good of the whole. This may be on the basis of supply chain integration, or general service streamlining through consolidation of applications and systems. Both objectives apply to the BBC Data Integration Service, for which this author serves as Delivery Manager on behalf of Siemens Business Services.

The Data Integration Service (DIS) has created an enterprise data model for media asset management (MAM), called the Standard Media Exchange Framework (SMEF), and a range of operating guidelines which build towards data interoperability across the corporation. DIS has been working with projects over the last few years to build compliance into systems which support different areas of the business. Each uses a different sub-set of SMEF with a core of commonality, so that where systems need to interconnect or exchange data they do so with consistent definitions and reference data sets. This is a longterm strategy, but can give immediate benefit to individual projects by avoiding the "clean sheet of paper" starting position. DIS also considers media interoperability and has devised a way of modeling media architecture which enables the overall environment to be understood and designed.

\section{BENEFITS OF A STRUCTURED APPROACH}

There are direct benefits of using a structured approach providing that the business commits to taking advantage of them. As stated above, there are benefits for project and system development through the reuse of knowledge and specifications, and it makes solution selection more robust if there is a clear expression of data requirements. It ensures that all users get the data they need to do their jobs, and buyers avoid falling into or perpetuating the sorts of problems summarized in the earlier examples.

In development, the work to connect or configure systems and make them interoperate with each other is made easier when they speak the same language, as it were. This is also relevant over time, enabling incremental system 
development rather than having to connect everything up at once in a Big Bang implementation. If the logical interoperability provided by an enterprise data architecture is also supported by a physical interoperability solution based on open standards, component technology or messaging, then significant future-proofing and flexibility can be achieved.

Media businesses should consider how best to approach data for themselves, based on the scale of their ambitions, the maturity of their technology service provision, and their ability to change working practices. The benefits of data integration to a media business embarking on DAM as an element of supply chain integration or global trading should be realized in process improvement and new opportunities. By making a broader range of information available to users without them having to search multiple systems or re-key and copy between applications, it should make them more able to respond quickly and appropriately to business demands. Staff and customers should be able to find the media and the information about it easily, and be able to understand and trust the information when they find it. It becomes easier to mount new services, and support existing ones more efficiently and effectively.

Technology and data integration are the enablers - the benefits can only be realized by the users and their managers, with support from their service providers. Media assets are only valuable if the information about them permits their reuse, so data in the DAM equation turns value into a definite plus. No data $=$ no value! 